\title{
A Prospective Study to Evaluate the Efficacy of Supersaturated Calcium Phosphate Oral Rinse in Patients with Xerostomia
}

\section{Rajiv Saini}

Department of Interdisciplinary Medicine, School of Medicine, University of Bari Aldo Moro, Bari, Italy

*Corresponding author: Rajiv Saini, Department of Interdisciplinary Medicine, School of Medicine, University of Bari Aldo Moro, Bari, Italy, Tel: +91-9923206789; Email: drrajivsaini@gmail.com

Received date: November 23, 2018; Accepted date: November 30, 2018; Published date: December 03, 2018

Copyright: (c) 2018 Saini R. This is an open-access article distributed under the terms of the Creative Commons Attribution License, which permits unrestricted use, distribution, and reproduction in any medium, provided the original author and source are credited.

\begin{abstract}
Background and Objective: Xerostomia exhibits symptoms of variable severity that are commonly attributed to medication use, chronic disease and medical treatment, such as radiotherapy to the head and neck region. Chronic xerostomia significantly increases the risk of experiencing dental caries, periodontal disease, and other oral diseases that may negatively affect the quality of life. This study was aimed to evaluate the beneficial effects of a Supersaturated Calcium Phosphate Rinse (SSCPR) in patients diagnosed with xerostomia.
\end{abstract}

Material and Methods: 38 subjects with xerostomia and high Streptococcus mutans levels were divided blindly under the control and test group. The control group received standard customary treatment while subjects under the test group were further advised to rinse with SSCPR 4 times per day for 3 months. Clinical, microbiological, and biochemical oral health parameters were evaluated after 3 months. The results were subjected to statistical analysis.

Results: Subjects under the test group i.e., using SSCPR had a 19\% reduction in gingival inflammation, a $16 \%$ reduction in plaque level and a $29 \%$ improvement in oral hygiene as compared to the control group. There was a $39 \%$ improvement in salivary flow and a more neutral $\mathrm{pH}$ in the oral cavity compared to the control group. The $S$. mutans load in the test group was markedly less than as compared to the control group. All outcomes demonstrated a statistically significant difference $(p<0.05)$.

Conclusion: Standard preventive and customary treatment when supplemented with SSCPR or SalivaMAX gave a statistically significant improvement in oral health for all outcomes measured (inflammation, plaque, oral hygiene, salivary flow, oral $\mathrm{pH}$, and $S$. mutans) when used regularly for a 3 month time period. SSCRP shows promise as a daily oral rinse and should be further evaluated for additional health benefits.

Keywords: Xerostomia; Hyposalivation; Saliva MAX; Streptococcus mutans, Caries risk; Oral health; Biofilm

Abbreviations: SSCPR: Supersaturated Calcium Phosphate Rinse; MST: Modified Schirmer Strip Test; GI: Gingival Index; PI: Plaque Index; OHI: Oral Hygiene Index

\section{Introduction}

The oral cavity is a barrier to systemic diseases and acts as an entry threshold for various infections that have the potential to compromise the immune system. The oral cavity is capable of harboring at least 600 different bacterial species, and in any given patient, more than 150 species may be present. Tooth surfaces alone can have as many as a billion bacteria attached to plaque formation; hence, good oral hygiene is fundamental for oral integrity and better quality of life. Recent advances in research technology have allowed researchers to study bacteria in their natural environment. Dental biofilm formation occurs through an ordered sequence of events, resulting in structured and functionally organized species-rich in microbial communities. Modern molecular biological techniques have identified about 1,000 different bacterial species in the dental biofilm. Dental plaque biofilm cannot be eliminated; however, the pathogenic nature of the dental plaque biofilm can be reduced by reducing the bio-burden (total microbial load and different pathogens isolated within dental plaque biofilm) and maintaining a normal flora with appropriate oral hygiene methods [1]. S. mutans bacteria formation intensifies in acidic intraoral environments resulting in a heightened risk for dental caries.

Periodontitis is a destructive inflammatory disease of the supporting tissues surrounding the teeth caused by specific microorganisms or groups of specific microorganisms resulting in progressive destruction of the periodontal ligament and alveolar bone with periodontal pocket formation, gingival recession, or both. The host responds to the periodontal infections with an array of events involving both innate and adaptive immunity [2]. Periodontitis has been proposed to have an etiological or modulating role in cardiovascular, cerebrovascular disease, diabetes, respiratory disease, and adverse pregnancy outcomes; several mechanisms have been proposed to explain or support such theories. Periodontal diseases are infectious processes that require bacterial presence and a host response and are affected by other local, environmental, and genetic factors. The host responds to the periodontal infections by various reactions involving both innate and adaptive immunity.

Salivary $\mathrm{pH}$ is the measurement of acidity or alkalinity of the saliva. The average intra-oral $\mathrm{pH}$ is around $\mathrm{pH} 7.4$ and is known to slightly decrease during the night. The average $\mathrm{pH}$ of normal saliva indicative of a healthy dental and periodontal environment ranges from 6.5 to 
7.5. At such a $\mathrm{pH}$, there is a low incidence of dental decay, whereas, decreased salivary $\mathrm{pH}$ increases susceptibility for enamel demineralization. Saliva, especially at a higher salivary $\mathrm{pH}$, acts as a buffer, facilitates remineralization of enamel, and minimizes aciduric bacteria. Bicarbonate, the main buffering component in saliva, neutralizes acids in the oral cavity and prevents changes in intra-oral $\mathrm{pH}$. Reduced or absent salivary function may result in decreased intraoral $\mathrm{pH}$ due to a compromised buffering capacity of the saliva. Additionally, low $\mathrm{pH}$ and a reduced salivary flow rate compromise the inflow rate of calcium and phosphate ions, which increases the risk of dental caries and demineralization.

Xerostomia is one of the most common debilitating conditions that impact Oral Health-Related Quality of Life (OHRQoL). Deficiency or absence of saliva cause significant morbidity and lead to the reduction of a person's quality of life. Oral dryness is one of the most common and most unpleasant oral symptoms which adversely affect all oral functions and compromise oral health. The decrease in salivary output creates an ionic instability primarily with calcium, phosphates, and bicarbonates which reduces the saliva's ability to function properly. Dry mouth leads to numerous oral sequelae including mucosal dryness, difficulty in chewing, swallowing and speaking, burning and pain of oral mucosa, the propensity of damage to oral mucosa and infection, increased fungal infection, demineralization of teeth and an increase in caries, dysgeusia, halitosis and difficulty in wearing dentures. Therefore, to maintain good oral and general health, saliva should be secreted in an adequate quantity and quality [3]. Decreased salivary flow due to reduced salivary function is called hyposalivation and the symptom of oral dryness is called xerostomia.

Xerostomia may affect individuals of all age groups, has many aetiologies, varies in degrees of severity, and contributes to the onset of both minor and major issues. Aetiologies of dry mouth include medication use, autoimmune diseases, hormonal changes, head and neck radiation, HIV disease, hepatitis C, Parkinson's disease, neoplasms, nerve damage, and many other conditions. Greater than 500 systemic medications are known to cause dry mouth. Sjogren's syndrome, a chronic inflammatory autoimmune disease, affects the salivary, lacrimal, and exocrine glands associated with the onset of dry mouth. Additionally, dry mouth is a side effect of various medical treatments. In the head and neck radiation patient population, studies have shown nearly $93 \%$ suffer from dry mouth, and $85 \%$ suffer from severe dry mouth. Dry mouth is a contributing factor to the onset of periodontal disease and tooth demineralization. Over time if left untreated, xerostomia is a gateway for a decrease in intra-oral $\mathrm{pH}$, an increase in plaque development and build-up, dental caries, and oral candidiasis. Salivary flow in the oral cavity has mineral content of fluoride, calcium, and phosphate and plays a crucial protective function for both soft and hard tissues. A saliva is a tool for cleaning the oral cavity, facilitating the process of chewing and swallowing, protecting oral tissue against physical and microbial harm, maintaining a neutral $\mathrm{pH}$, and preventing demineralization of tooth surfaces. Salivary flow is supplied by 3 major salivary glands; the parotid, the submandibular, and the sublingual in addition to the minor salivary glands distributed throughout the oral cavity. The respective estimated flow rates are $65 \%$ or $0.26 \mathrm{~mL} / \mathrm{min}$ from the parotid gland, $20-30 \%$ or $0.08 \mathrm{~mL} / \mathrm{min}$ from the submandibular, $6 \%$ or $0.03 \mathrm{~mL} / \mathrm{min}$ from the sublingual, and $5 \%$ or $0.03 \mathrm{~mL} / \mathrm{min}$ from the minor salivary glands. Normal daily secretion of saliva is approximately $1-1.5 \mathrm{~L}$ per day $(0.5-1 \mathrm{~mL} / \mathrm{min})$ in the fluctuations due to diurnal variation, hydration, food intake, exercise, and many other factors. A large variability in salivary flow is reported among individuals, and thus, a standard value is not explicitly defined. Generally, though, an unstimulated salivary flow of $0.1 \mathrm{~mL} / \mathrm{min}$ (measured for 5-15 minutes) and a stimulated salivary flow of 0.5 $\mathrm{mL} / \mathrm{min}$ are indicative for salivary hyposecretion or hypofunction [4].

Efforts have been made recently to reduce the effects of xerostomia. This study was aimed to evaluate the beneficial effects of an SSCPR (SalivaMAX, Forward Science, USA) in the oral cavity of patients diagnosed with xerostomia.

\section{Material and Methods}

This randomized, double-blind clinical study was coordinated by Innovative Biological Research Centre, Pune, India. Patients received a verbal description of the clinical protocol to be followed in this study. The subjects selected for the study were educated regarding the study being carried out and informed consent was obtained. Subjects were assigned based on a computer-generated random allocation system to either the investigational group or the control group. Both groups were given an exam and standard preventative customary treatment and symptomatic treatment when required. The investigational group rinsed with an SSCPR 4 times per day for a 3 month period in addition to the standard treatment. Other than the preventative customary treatment and symptomatic treatment, no supplementary therapeutic/ prescription rinse was advised for the control group.

Subjects were enrolled with fixed inclusion and exclusion criteria as illustrated in Table 1 . The primary inclusion criteria were xerostomia with moderate to severe $S$. mutans colonization. The subject population was reduced for not meeting inclusion/exclusion criteria, lost to follow-up/voluntary withdrawal, or non-compliance; 38 subjects, 19 in each arm, completed the study and were available for final evaluation (Figure 1).

Patients were initially screened for their hyposalivation condition using the Modified Schirmer Strip Test (MST) [5]. Those 62 subjects having an MST score $<25 \mathrm{~mm}$ at 3 minutes, which is indicative of hyposalivation/xerostomia, were selected for this study. Salivary samples were taken from the 62 subjects to quantify $S$. mutans levels as illustrated in Table 2. A total of 53 subjects fell under the Class II or Class III and further met the inclusion criteria. During the recruitment process, five subjects withdrew from the study, leaving 48 subjects for randomization. During the study, seven subjects voluntarily withdrew from the study and three subjects failed to follow the study protocol instruction and were excluded from the evaluation criteria. The result was 38 subjects available for analysis with 19 subjects in each arm.

Base line clinical measurements of Gingival Index (GI) [6], Plaque Index (PI) [7], and Oral Hygiene Index (OHI) [8] were recorded. Biochemistry parameters of the MST (Bell Pharma, Mumbai, India) and potential of Hydrogen, $\mathrm{pH}$, (Forward Science, Texas, USA) were recorded for both the groups. The microbiology measurements (i.e., $S$. mutans level) were also recorded for both the groups. Recall visits were scheduled for all the subjects at 3 months from baseline and all measurements were recorded again. A t-test was used for analysis in all measures, except for the $S$. mutans level which used Fisher's Exact Test. 
Citation: Saini R (2018) A Prospective Study to Evaluate the Efficacy of Supersaturated Calcium Phosphate Oral Rinse in Patients with Xerostomia. Dentistry 8: 522. doi:10.4172/2161-1122.1000522

Page 3 of 5

\begin{tabular}{|c|c|}
\hline \multirow[b]{6}{*}{ Inclusion criteria } & Adult population (18 years and above) \\
\hline & Presence of all lower arch (Mandible) anterior teeth \\
\hline & Subjects with Modified Schirmer Test (MST) value $<25 \mathrm{~mL} / 3$ minute \\
\hline & Subjects that meet the salivary S. mutans colonization with moderate to severe Colony Forming Units (CFUs) \\
\hline & Willing to participate voluntarily in the study \\
\hline & The patient should have a minimum of 20 sound permanent teeth with a minimum of 5 teeth to be present in each arch quadrant \\
\hline \multirow[b]{8}{*}{ Exclusion criteria } & Presence of any systemic neurological disorder (e.g., epilepsy or schizophrenia) \\
\hline & Presence of a disease with possible effects on the immune system \\
\hline & Patient on active treatment lined up any nearby medical procedure or surgery \\
\hline & Patients currently on periodontal treatment/oral surgical treatment \\
\hline & Pregnant and lactating mother \\
\hline & No active chemotherapy/radiation \\
\hline & Inability to use mouthwash \\
\hline & Subjects not willing to participate in the study \\
\hline
\end{tabular}

Table 1: Study inclusion and exclusion criteria.

The SSCPR evaluated during this study was SalivaMAX, an FDA cleared product from Forward Science, Texas, USA. SalivaMAX is a prescription strength rinse that is indicated for the relief of oral mucositis and xerostomia, also known as dry mouth. SalivaMAX is artificial saliva composed of calcium, phosphate, bicarbonate and chloride electrolytes that is provided in single-use disposable packets. The goal of administering SalivaMAX is to relieve chronic and temporary xerostomia and mucositis.

\begin{tabular}{|l|l|l|}
\hline Class & CFU/mL & Caries susceptibility \\
\hline Class 0 & $<10^{3}$ & Negligible \\
\hline Class I & $10^{3}-10^{4}$ & Low \\
\hline Class II & $10^{4}-10^{5}$ & Moderate \\
\hline Class III & $>10^{5}$ & High \\
\hline
\end{tabular}

Table 2: Categorization based on $S$. mutans levels.

\section{Results}

The total number of subjects enrolled and analyzed was 38 . In the SSCPR group, there were 9 males and 10 female subjects with an average age of $43.8 \pm 4.9$. In the control group, there were 10 males and 9 female subjects with an average age of $44.6 \pm 6.7$. No adverse events were reported.

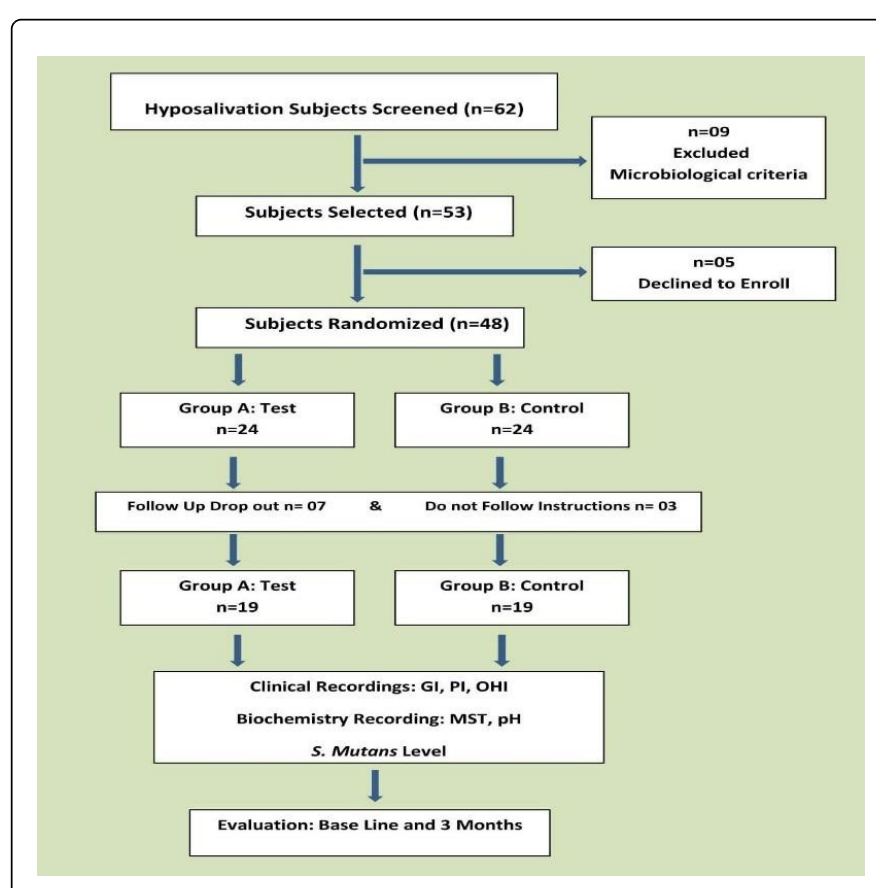

Figure 1: Study flow chart.

At baseline, all the parameters (GI, PI, OHI, MST, pH, S. mutans category) evidenced the two groups were not statistically different ( $>0.05$ ) demonstrating suitable randomization of the populations. The baseline GI of the two groups, a mean of about 2.7, indicated that the baseline grade of inflammation was severe. The PI of the two groups, a mean of about 2.6, indicated poor plaque control. The baseline $\mathrm{OHI}$ of the two groups had a mean of about 3.5 representing 
oral hygiene in poor condition with an abundance of debris and calculus. Further details are provided in Table 3. The mean value of MST in both groups at baseline was about 19 which was considered xerostomic. The mean value of $\mathrm{pH}$ in both groups was approximately 5.8, defining an acidic environment in the oral cavity. As part of the inclusion criteria, all subjects at the baseline were a Class II or Class III S. mutans grade (Figure 2a) indicating that all the subjects in both groups were susceptible to moderate to high caries risk at the initiation of the study.

\begin{tabular}{|c|c|c|c|c|c|c|}
\hline & \multicolumn{2}{|c|}{ Gingival Index (GI) } & \multicolumn{2}{|c|}{ Plaque Index (PI) } & \multicolumn{2}{|c|}{ Oral Hygiene Index (OHI) } \\
\hline & Baseline $^{*}$ & 3 months $\S$ & Baseline $^{*}$ & 3 months $\S$ & Baseline $^{*}$ & 3 months $\S$ \\
\hline SSCPR & $2.72( \pm 0.28)$ & $0.78( \pm 0.10)$ & $2.55( \pm 0.25)$ & $0.82( \pm 0.11)$ & $3.46( \pm 0.26)$ & $1.16( \pm 0.31)$ \\
\hline Control & $2.65( \pm 0.29)$ & $1.26( \pm 0.08)$ & $2.70( \pm 0.20)$ & $1.31( \pm 0.12)$ & $3.53( \pm 0.27)$ & $2.20( \pm 0.35)$ \\
\hline
\end{tabular}

Parentheses indicate standard deviation

*No statistically significant difference $(p>0.05)$ at baseline between the SSCPR and control groups

$\S$ There was a statistically significant difference $(p<0.05)$ between the SSCPR and control groups for the outcome at the 3 months follow up

Table 3: Study measurement parameters.

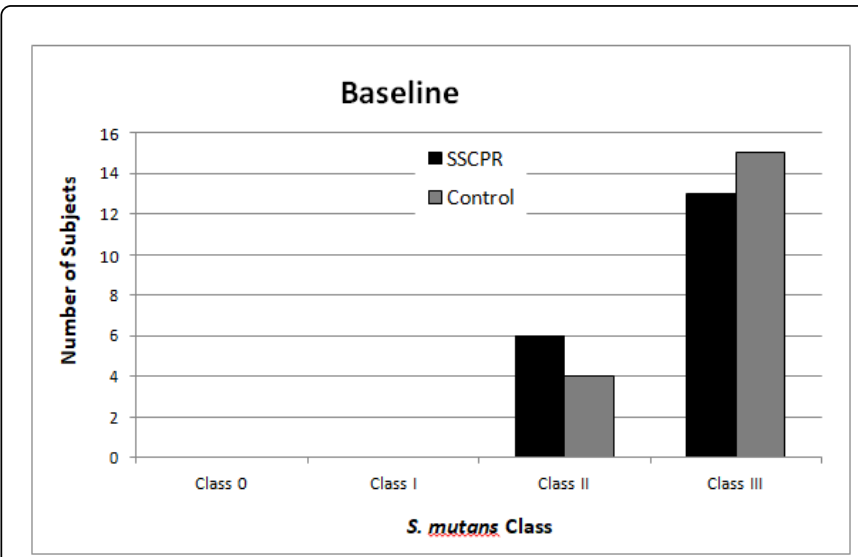

Figure 2a: $S$. mutans class for both groups at the baseline. Note that at the baseline only Class II and Class III $S$. mutans levels were enrolled in the study. There was no statistically significant difference $(\mathrm{p}>0.05)$ between the two groups at baseline.

The GI score in the SSCPR group at 3 months was $0.78 \pm 0.10$ indicating only mild inflammation while the control group was $1.26 \pm$ 0.08 denoting moderate inflammation. The mean value of PI in the SSCPR group at 3 months was $0.82 \pm 0.11$ representing good/ acceptable plaque control while the control group PI was $1.31 \pm 0.12$ signifying only fair plaque control. The mean value of $\mathrm{OHI}$ at 3 months in the SSCPR group was $1.16 \pm 0.31$ indicating good/acceptable oral hygiene condition while the control group was $2.20 \pm 0.35$ denoting only a fair condition. The mean value of MST in the SSCPR group at 3 months was $32.47 \mathrm{~mm} \pm 0.96$ indicating saliva flow above the xerostomic threshold while the control group was $26.10 \mathrm{~mm} \pm 1.91$ and considered just above the threshold for xerostomia, $25 \mathrm{~mm}$. The mean value of $\mathrm{pH}$ in the SSCPR group at 3 months was $7.26 \pm 0.25$ representing salivary $\mathrm{pH}$ in a healthy range while the control group $\mathrm{pH}$ was $6.5 \pm 0.33$ denoting a salivary $\mathrm{pH}$ just at the threshold of an acidic and neutral oral cavity. Figure $2 \mathrm{~b}$ demonstrates the dramatic reduction of $S$. mutans grade at 3 months for the SSCPR group over the control.

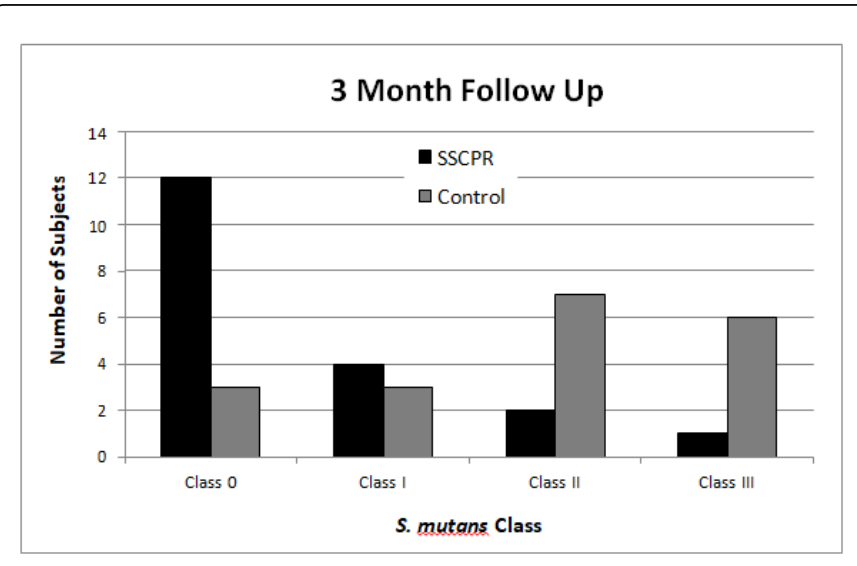

Figure 2b: $S$. mutans class for both groups at the 3 months follow up. There was a statistically significant difference $(\mathrm{p}<0.05)$ between the SSCPR and control groups at the 3 months follow up.

Both the SSCPR and control groups had a statistically significant improvement in all scores at the 3 months follow up as compared to the baseline demonstrating the value of standard preventative treatment and symptomatic treatment both groups revived. However, in addition to the improvement that the preventative customary treatment and symptomatic treatment provided, the SSCPR group showed a marked and statically significant improvement over the control group at 3 months in all outcomes (inflammation, plaque control, oral hygiene control, saliva flow, and $\mathrm{pH}$ ) indicating the SSCPR provided an additional level of hygiene and potential benefits to the patients' oral health and quality of life.

\section{Discussion}

The present clinical study was undertaken to evaluate and compare the effects of an SSCPR (SalivaMAX) in patients diagnosed with Xerostomia/hyposalivation compared to the standard preventive customary treatment for xerostomia on oral health. For all measures, the SSCPR group demonstrated a statistically significant $\mathrm{p}<0.05$ improvement in the measured parameters over the control group at the 3 months follow up. 
The GI score at 3 months in the SSCPR group demonstrated a 19\% increased reduction in gingival inflammation over the control group. This statistically significant difference proved the beneficial effects of the SSCPR (SalivaMAX) in lowering the gingival and periodontal inflammatory response. By controlling the gingival inflammation, the possibility of further periodontal destruction is likely to be minimized further promoting oral health.

The PI at 3 months in the SSCPR group demonstrated a $16 \%$ greater reduction in plaque over the control group. To control plaque accumulation (i.e., biofilm formation) in the oral cavity is always challenging; this becomes more difficult and detrimental to oral health as calculus is formed. By controlling the dental plaque at a nascent stage, clinicians can promote a long-term beneficial effect especially when the patient is compromised like in a xerostomic condition. SSCPR proved to be a potent tool in restoring plaque level compared to the control group.

The OHI at 3 months in the SSCPR group established a $29 \%$ greater improvement in oral hygiene over the control group. These results showed the potential benefits of SSCPR (SalivaMAX) for the control of deposition of debris and calculus formation and thus maintained overall oral hygiene at a good level as compared to the control group who had fair oral hygiene only.

The MST in the SSCPR group at 3 months showed a 39\% greater improvement in hyposalivation over the control group. While it is generally understood that the SSCPR adds ions to the oral cavity which are at a low concentration or even missing in xerostomic patents, the data suggest that natural saliva may be increased with the use of the SSCPR.

The value of salivary $\mathrm{pH}$ in the SSCPR group at 3 months was 0.76 higher than the control group, 7.26 vs 6.5 , respectively. A normal salivary $\mathrm{pH}$ (6.5-7.5) usually indicates a healthy dental and periodontal condition. At this normal $\mathrm{pH}$, there is a low incidence of dental decay and little or no calculus. The decrease in salivary $\mathrm{pH}$ increases the susceptibility towards enamel demineralization. The SSCPR group maintained a healthy $\mathrm{pH}$ of 7.2 compared to the control group that averaged the marginal healthy $\mathrm{pH}$ of 6.5 . The significant difference between the SSCPR and control groups evidences the potential benefits of boosting oral health by means of supplemental treatment using SSCPR.

In the SSCPR group, only 3 subjects classified as Class II or Class III score, while the control group had 13 in the Class II or Class III category at 3 months. This difference was statistically significantly different $(p<0.05)$ using Fisher's Exact Test. Figure $2 b$ shows the dramatic shift in $S$. mutans levels at the 3 months follow up for the SSCPR group; the control group has a markedly and statically significant higher carries risk than the SSCPR group at the 3 months follow up.

\section{Conclusion}

Xerostomia is a chronic condition induced by many aetiologies such as head and neck radiation, autoimmune conditions, and various systemic medications. Additionally, xerostomia is a gateway to the onset of significant oral health sequelae. Primarily, low $\mathrm{pH}$ and low calcium and phosphate concentrations will lead to demineralization [9]. Such a state combined with a high $S$. mutans level confirms caries is a very real risk [10] that remains as long as the xerostomic conditions exist [11] reported that continued use of an SSCPR in a high-risk (xerostomic) population was protective against caries progression.

Standard preventative and customary treatment supplemented with an SSCPR (SalivaMAX) demonstrated a statistically significant improvement in the GI score, PI score, OHI score, MST, $\mathrm{pH}$, and $S$. mutans level in a xerostomic population. Based on the results of this study, the oral hygiene of a xerostomic population can be markedly improved with continued use of an SSCPR. An SSCRP shows promise as a daily oral rinse and should be further evaluated for additional health benefits. However, confirmation of this study requires a longterm multi-centric clinical trial for outcomes of SSCPR (SalivaMAX) under different hyposalivation conditions.

\section{Acknowledgment}

None

\section{References}

1. Saini R, Saini S, Sharma S (2011) Biofilm: A dental microbial infection. J Nat Sc Biol Med 2: 71-75.

2. Saini R, Saini S, Saini SR (2010) Periodontal diseases: a risk factor to cardiovascular disease. Ann Card Anaesth 13: 159-161.

3. In: Sreebny LM, Vissink A (eds.) (2010) Dry mouth, the malevolent symptom: a clinical guide. Wiley-Blackwell, pp: 1-268.

4. Navazesh M, Christensen C, Brightman V (1992) Clinical criteria for the diagnosis of salivary gland hypofunction. J Dent Res 71: 1363-1369.

5. Chen A, Wai Y, Lee L, Lake S, Woo SB (2005) Using the modified Schirmer test to measure mouth dryness: a preliminary study. J Am Dent Assoc 136: 164-170.

6. Loe H, Silness J (1963) Periodontal disease in pregnancy. I Prevalence and severity. Acta Odont Scand 21: 533-551.

7. Silness J, Loe H (1964) Periodontal disease in pregnancy. II. Correlation between oral hygiene and periodontal condition. Acta Odontol Scand 22: 121-135.

8. Greene JC, Vermillion JR (1960) The oral hygiene index: a method for classifying oral hygiene status. J Am Dent Assoc 61: 172-179.

9. Dawes C (2003) What is the critical pH and why does a tooth dissolve in acid? J Can Dent Assoc 69: 722-724.

10. Papas AS, Joshi A, MacDonald SL, Maravelis-Splagounias L, PretaraSpanedda P, et al. (1993) Caries prevalence in xerostomic individuals. J Can Dent Assoc 59: 171-174.

11. Singh ML, Papas AS (2009) Long-term clinical observation of dental caries in salivary hypofunction patients using a supersaturated calciumphosphate remineralizing rinse. J Clin Dent 20: 87-92. 